\title{
A Brief History of the Stringy Instanton
}

\author{
Nick Dorey ${ }^{a}$, Timothy J. Hollowood ${ }^{a}$ and Valentin V. Khoze ${ }^{b}$ \\ ${ }^{a}$ Department of Physics, University of Wales Swansea, Swansea, SA2 8PP, UK \\ ${ }^{b}$ Department of Physics and IPPP, University of Durham, Durham, DH1 3LE, UK \\ E-mail: n.dorey@swan.ac.uk, t.hollowood@swan.ac.uk, valya.khoze@durham.ac.uk
}

ABstract: The arcane ADHM construction of Yang-Mills instantons can be very naturally understood in the framework of D-brane dynamics in string theory. In this point-of-view, the mysterious auxiliary symmetry of the ADHM construction arises as a gauge symmetry and the instantons are modified at short distances where string effects become important. By decoupling the stringy effects, one can recover all the instanton formalism, including the all-important volume form on the instanton moduli space. We describe applications of the instanton calculus to the AdS/CFT correspondence and higher derivative terms in the D3-brane effective action. In these applications, we are starting to uncover an interesting relation between instanton partition functions, the Euler characteristic of instanton moduli space and modular symmetry. We also describe how it is now possible to do multi-instanton calculations in gauge theory and we resolve an old puzzle involving the gluino condensate in supersymmetric QCD.

KEYwords: IInstantons, D-branes, AdS/CFT correspondence, Euler characteristió.

\section{Introduction}

There has been a wealth of progress in understanding semi-classical effects in supersymmetric gauge theories in the last few years. In this review we shall be concentrating on instantons and one of the main goals is show how naturally Yang-Mills instantons appear in string theory.

Instantons are solutions of the classical equations of gauge theory with finite action. Charge $k$ instanton effects come along with a factor

$$
\begin{aligned}
& \exp \left(-\frac{1}{2 g^{2}} \int F \wedge^{*} F+\frac{i \vartheta}{16 \pi^{2}} \int F \wedge F\right) \\
& =\exp \left(-\frac{8 \pi^{2} k}{g^{2}}+i k \vartheta\right) \equiv e^{2 \pi i k \tau} \equiv q^{k} \sim \Lambda^{k b_{1}},
\end{aligned}
$$

where $b_{1}$ is the first coefficient of the beta function. Certain quantities in SUSY gauge theory are protected by powerful non-renormalization theorems or "holomorphy". In these cases, instanton contributions are exact in the sense that there are no perturbative corrections since these would involve a series in the non-holomorphic quantity $g^{2}$. The classic example of such a protected quantity is the gluino condensate in $\mathcal{N}=1$
SUSY gauge theory: for gauge group $S U(N)$ an instanton calculation gives [1]

$$
\left\langle\frac{\lambda^{2}}{16 \pi^{2}}\right\rangle=\frac{2 \Lambda^{3}}{[(N-1) !(3 N-1)]^{1 / N}} .
$$

Actually this appears to be a charge $k=\frac{1}{N}$ effect based on the power of $\Lambda$, so what is calculated is the instanton contribution to the $\mathrm{N}$-point function which is independent of the insertion points by a SUSY Ward identity. Clustering is then invoked (taking into account averaging over the $N$ physically equivalent vacua of the theory) to extract (1.21).

Unfortunately, we have recently learned that this procedure does not give the correct answer for the gluino condensate [2]. ${ }^{1}$ Suspicion should have been aroused from the start, after all an instanton calculation is a semi-classical method, whereas the theory in question is in a stronglycoupled confining phase. So although there are

\footnotetext{
${ }^{1}$ One of the reasons for believing in the result is due to a topological field theory style argument along the lines that the semi-classical approximation should be exact. However, this only applies to the theory on a compact spacetime, like the torus and not on $\mathbb{R}^{4}$.
} 
no perturbative corrections around the instanton contribution there can be other non-perturbative, but non-instanton, contributions. How can we be sure that other configurations contribute to the gluino condensate? We know this because in [2] we calculated for the first time multi-instanton contributions to multi-point functions of the gluino and showed that clustering is violated: a sure sign that other configurations must contribute. Specifically, we calculated the contribution of $k$ instantons to the $k N$-point function, at large $N$ (which is enough to prove the point), and showed in this limit

$$
\left\langle\frac{\lambda^{2}\left(x_{1}\right)}{16 \pi^{2}} \times \cdots \times \frac{\lambda^{2}\left(x_{k N}\right)}{16 \pi^{2}}\right\rangle^{\frac{1}{k N}} \propto k,
$$

instead of being independent of $k$, if clustering was respected in the instanton sector.

The message of this work is two-fold: firstly we have demonstrated that multi-instanton calculations are now technically feasible, particularly in the large $N$ limit where a number of important simplifications occur. Secondly, instanton calculations will be valid only in a weaklycoupled phase. This last point looks rather restrictive; however, this is not the case and one can infer the value of a quantities like the gluino condensate in a strongly coupled phase using a two-stage procedure.

The idea is to modify the theory so that there is a new coupling constant which in some limit drives the theory into weak coupling, but which in the opposite limit returns one smoothly to the confining phase. Under suitable circumstances the gluino condensate will be holomorphic in this new coupling and the weak-coupled result can be analytically continued to strong coupling. There are at least two ways to achieve this and both yield the correct answer for the gluino condensate

$$
\left\langle\frac{\lambda^{2}}{16 \pi^{2}}\right\rangle=\Lambda^{3}
$$

Method 1. [3] Add matter fields to completely break the gauge group. For example, we can add $N-1$ chiral multiplets in the $\boldsymbol{N}+\overline{\boldsymbol{N}}$. The resulting theory is in a weakly-coupled Higgs phase for small values of the masses. When the masses go to infinity, the matter fields decouple and the theory is continuously connected to the confining phase of the pure gauge theory. The gluino condensate can now be calculated reliably. In this case the 1-point function is a oneinstanton effect.

Method 2. [4] Put the theory ${ }^{2}$ on a cylinder $\mathbb{R}^{3} \times S^{1}$. In this case, the gauge field can have a Wilson line around the $S^{1}$. This breaks the gauge group to $U(1)^{r}$ and so, generically, the theory is in a Coulomb phase. For small value of the radius the theory is weakly coupled and the gluino condensate can be calculated reliably. The result can then be continued to large $R$ and gives the value of the gluino condensate in the uncompactified theory. Interestingly, on the cylinder the topological charge is not constrained to be integer and there are other finite action configurations which arise as monopoles in the gauge theory whose world-lines wrap the $S^{1}$. The gluino condensate now receives contributions from monopoles. For a general gauge group there are $r$ fundamental monopoles (those which are not composite configurations) whose magnetic charges are proportional to the co-simple roots $\boldsymbol{\alpha}_{i}^{*}$ of $\mathfrak{g}$. However, on the cylinder there is an additional solution, the "affine" monopole, whose magnetic charge is the lowest co-root $\boldsymbol{\alpha}_{0}^{*}$ [5]. This solution is special to the cylinder since it depends non-trivially on the coordinate around the circle. Amazingly, an instanton in the theory on the cylinder is a composite configuration consisting of one of each of the $r+1$ fundamental monopoles. For small radius the theory is in a weakly-coupled Coulomb phase and the fundamental monopole contribute to a superpotential in the low energy effect action of the 3-dimensional $U(1)^{r}$ gauge theory. This superpotential depends on an $r$-vector superfield $\boldsymbol{X}$ whose scalar component is

$$
\boldsymbol{\sigma}+\tau \varphi
$$

where $\boldsymbol{\sigma}$ is the dual $U(1)^{r}$ gauge field and $\boldsymbol{\varphi}$ is the Wilson line. The superpotential has the form of an affine Toda potential for $\left(\mathfrak{g}^{(1)}\right)^{*}$, matching calculations via $\mathrm{M}$ theory [6]:

$$
W(\boldsymbol{X}) \sim \sum_{j=1}^{r} \frac{2}{\boldsymbol{\alpha}_{j}^{2}} e^{i \boldsymbol{\alpha}_{j}^{*} \cdot \boldsymbol{X}}+q \frac{2}{\boldsymbol{\alpha}_{0}^{2}} e^{i \boldsymbol{\alpha}_{0}^{*} \cdot \boldsymbol{X}} .
$$

${ }^{2}$ This method works for and arbitrary gauge group $G$, with Lie algebra $\mathfrak{g}$, and we will take $r$ to be the rank. 
where $\boldsymbol{\alpha}^{*}=2 \boldsymbol{\alpha} / \boldsymbol{\alpha}^{2}$ are the dual roots. This potential has $c_{2}$ SUSY vacua (in agreement with the Witten index) and the magnitude of the gluino condensate in each of the vacua is

$$
\left\langle\frac{\lambda^{2}}{16 \pi^{2}}\right\rangle=\frac{\Lambda^{3}}{\prod_{j=0}^{r}\left(k_{j}^{*} \boldsymbol{\alpha}_{j}^{2} / 2\right)^{k_{j}^{*} / c_{2}}},
$$

where $k_{j}^{*}$ are the dual Kac labels. Interestingly in each vacua the monopole carry a fraction $1 / c_{2}$ of topological charge which means that they realize the old idea that an instanton is made up of constituents.

The connection of superpotentials in SUSY gauge theories compactified on a cylinder and integrable theories is more general. The most remarkable example is the mass deformed $\mathcal{N}=4$ theory (or the $\mathcal{N}=1^{*}$ theory) [7]. The $\mathcal{N}=4$ theory consists of an $\mathcal{N}=1$ vector multiplet and 3 adjoint-valued $\mathcal{N}=1$ chiral multiplets, each of which can be given a mass $m_{1,2,3}$ which breaks $\mathcal{N}=4 \rightarrow 1$. For gauge group $S U(N)$, the superpotential for the theory compactified on the cylinder is the complexified potential of the $N$ body elliptic Calogero-Moser system:

$$
W(\boldsymbol{X}) \sim m_{1} m_{2} m_{3} \sum_{\boldsymbol{\alpha}} \wp(\boldsymbol{\alpha} \cdot \boldsymbol{X}) .
$$

When expanded in $q$, terms can be identified with particular configurations involving monopoles and instantons [7]. This superpotential has some remarkable modular properties and one can extract a wealth of information from it [8].

\section{Multi-Instantons and ADHM}

We now need to get to grips with the calculus of multi-instantons on $\mathbb{R}^{4}$ as described by ADHM [10]. On first exposure, the ADHM construction looks rather ad-hoc; however in the following section we shall describe 2 ways to interpret it.

We start by considering a single instanton in $S U(N)$. This is constructed by taking an $S U(2)$ instanton, which has a scale size and position in $\mathbb{R}^{4}$, and then orientating it inside $S U(N)$, which involves $4 N-5$ additional "coset" parameters. Rather perversely, we want to describe these moduli in the following way: firstly $a_{n}^{\prime}$, which is (minus) the position of the instanton.
To this we add 2 complex $N$ vectors $w_{u \dot{\alpha}}$ subject to the 3 constraints

$$
\left(\tau^{c}\right)_{\dot{\beta}}^{\dot{\alpha}} \bar{w}^{\dot{\beta}} w_{\dot{\alpha}}=0
$$

(Obviously, we could easily solve the constraints in this case.) The instanton solution is actually independent of an auxiliary $U(1)$ which rotates $w_{\dot{\alpha}}$ by a phase. The physical meaning of the parameters is

$$
\begin{array}{ccc}
a_{n}^{\prime} & \longrightarrow & - \text { position in } \mathbb{R}^{4} \\
\rho^{2}=\bar{w}^{\dot{\alpha}} w_{\dot{\alpha}} & \longrightarrow & \operatorname{size}^{2} \\
\rho^{-2} w_{u \dot{\alpha}}\left(\tau^{c}\right)_{\dot{\beta}}^{\dot{\alpha}} \bar{w}_{v}^{\dot{\beta}} & \longrightarrow & S U(2) \subset S U(N)
\end{array}
$$

Multi-instantons are described by a non-abelian generalization of this construction. The instanton position $a_{n}^{\prime}$ becomes a 4 -vector of $k \times k$ hermitian matrices and there are $2 k N$-vectors $w_{u i \dot{\alpha}}$, $i=1, \ldots, k$. The generalization of $\left(\left[\begin{array}{l}2 \\ -1\end{array}\right)\right.$ is the famous set of ADHM constraints:

$$
\mathcal{B}^{c} \equiv\left(\tau^{c}\right)_{\dot{\beta}}^{\dot{\alpha}}\left(\bar{w}^{\dot{\beta}} w_{\dot{\alpha}}+\bar{a}^{\prime \dot{\beta} \alpha} a_{\alpha \dot{\alpha}}^{\prime}\right)=0,
$$

where $a_{\alpha \dot{\alpha}}^{\prime}=a_{n}^{\prime} \sigma_{\alpha \dot{\alpha}}^{n}$. The moduli space of $k$ instantons, $\mathfrak{M}_{k, N}$, is then given by $\left\{a_{n}^{\prime}, w_{\dot{\alpha}}\right\}$ modulo the ADHM constraints and modulo an auxiliary $U(k)$ symmetry which acts as $w_{\dot{\alpha}} \rightarrow w_{\dot{\alpha}} U$, $a_{n}^{\prime} \rightarrow U^{\dagger} a_{n}^{\prime} U$.

Before we leave this section, let us consider three important things.

(i) In a supersymmetric theory, instantons also have Grassmann moduli which arise from the fermion fields (see [3,11-13]). In a SUSY gauge theory with $\mathcal{N}$ supersymmetries $(\mathcal{N}=1,2,4)$, there are $\mathcal{N}$ gluino fields and the corresponding Grassmann collective coordinates are $k \times k$ matrices $\mathcal{M}_{\alpha}^{\prime}{ }_{\alpha}, k \times N$ matrices $\mu^{A}$ and $N \times k$ matrices $\bar{\mu}^{A}$, where $A=1, \ldots, \mathcal{N}$. These are subject to fermionic analogues of the ADHM constraints:

$$
\mathcal{F}_{\dot{\alpha}}^{A} \equiv \bar{\mu}^{A} w_{\dot{\alpha}}+\bar{w}_{\dot{\alpha}} \mu^{A}+\left[\mathcal{M}^{\prime \alpha}, a_{\alpha \dot{\alpha}}^{\prime}\right]=0 \text {. }
$$

(ii) In order to do instanton calculations, we need to known how to change variables in the path integral from the fields to the collective coordinates. A direct approach to this problem has only been achieved in the cases $k=1,2[14,15]$. An alternative and tractable approach [16] is to use the symmetries of the theory, and in this respect SUSY is a very powerful symmetry, along 
with cluster decomposition to deduce the measure on the SUSY instanton moduli space at arbitrary $k$. The resulting expression for the measure is fortunately rather simple:

$z_{k, N}=\int \frac{d^{4} a^{\prime} d^{2} w d^{2 \mathcal{N}} \mathcal{M}^{\prime} d^{\mathcal{N}} \mu d^{\mathcal{N}} \bar{\mu} \delta\left(\mathcal{B}^{c}\right) \delta\left(\mathcal{F}_{\dot{\alpha}}^{A}\right)}{\operatorname{Vol} U(k)(\operatorname{det} \boldsymbol{L})^{\mathcal{N}-1}}$

Here $\boldsymbol{L}$ is an operator on $k \times k$ matrices:

$$
\boldsymbol{L} \cdot \Omega=\left\{\bar{w}^{\dot{\alpha}} w_{\dot{\alpha}}, \Omega\right\}+\left[a_{n}^{\prime},\left[a_{n}^{\prime}, \Omega\right]\right] .
$$

Given the measure in any of the supersymmetric theories, the measure in QCD $(\mathcal{N}=0)$ can be deduced by giving masses to the fermions and then using renormalization group decoupling. Remarkably the resulting expression is also given by the formula (2.5). It is now possible to write down the $k$ instanton measure in pure QCD, including the one-loop fluctuation determinants, since these can be extracted from the old instanton literature [9]. Since, to our knowledge this has never been written down, we do it here

$$
z_{\mathrm{QCD}}=\int \frac{d^{4} a^{\prime} d^{2} w \delta\left(\mathcal{B}^{c}\right)}{\operatorname{Vol} U(k)} \cdot \operatorname{det} \boldsymbol{L} \cdot(\operatorname{det} \Delta)^{-2},
$$

where the fluctuation determinant is

$(\operatorname{det} \Delta)^{-2}=\mu^{-k N / 3}(\operatorname{det} \boldsymbol{L})^{-1} e^{-\frac{1}{24 \pi^{2}} \int d^{4} x\left(I_{1}+I_{2}+I_{3}\right)}$,

where $I_{c}(x)$ are all functions of the $k \times k$ matrix $f^{-1}(x)=\frac{1}{2} \bar{w}^{\dot{\alpha}} w_{\dot{\alpha}}+\left(a_{n}^{\prime}+x_{n} 1_{[k] \times[k]}\right)\left(a_{n}^{\prime}+x_{n} 1_{[k] \times[k]}\right)$,

given by

$$
\begin{gathered}
I_{1}(x)=\operatorname{tr}_{k}\left(f \partial_{n} f^{-1} f \partial_{n} f^{-1} f \partial_{m} f^{-1} f \partial_{m} f^{-1}\right. \\
\left.-20 f^{2}\right)+\frac{4 k}{\left(1+x^{2}\right)^{2}}, \\
I_{2}(x)=\int_{0}^{1} d t \epsilon_{m n p q} \operatorname{tr}_{k}\left(\tilde{f} \partial_{t} \tilde{f}^{-1} \tilde{f} \partial_{m} \tilde{f}^{-1}\right. \\
\left.\times \tilde{f} \partial_{n} \tilde{f}^{-1} \tilde{f} \partial_{p} \tilde{f}^{-1} \tilde{f} \partial_{q} \tilde{f}^{-1}\right), \\
I_{3}(x)=\log \operatorname{det} f \square^{2} \log \operatorname{det} f .
\end{gathered}
$$

Here, $t$ is an auxiliary variable and $\tilde{f}(x, t)$ is the $k \times k$ dimensional matrix derived from $f(x)$ :

$$
\tilde{f}^{-1}(x, t)=t f^{-1}(x)+(1-t)\left(1+x^{2}\right) 1_{[k] \times[k]} .
$$

In (2.7),$\mu$ is the mass parameter of the PauliVillars regularization scheme. Of course we should emphasize that QCD is not weakly coupled and in the light of our previous discussion, we should be rather careful in using the instanton approximation in this context.

In an $\mathcal{N}=4$ SUSY gauge theory the measure $(2.5)$ is not the complete story because in these theories the action evaluated on the instanton solution is not just the constant (11.1). In these theories all but the 8 SUSY and 8 superconformal fermion zero modes, which are protected by the corresponding symmetries, are lifted beyond linear order at the classical level by the Yukawa interactions of the theory [13]. This leads to a 4 -fermion term in the instanton action:

$$
\begin{aligned}
\frac{\pi^{2}}{2 g^{2}} \epsilon_{A B C D} & \operatorname{tr}_{k}\left(\bar{\mu}^{A} \mu^{B}+\mathcal{M}^{\prime \alpha A} \mathcal{M}_{\alpha}^{\prime B}\right) \\
& \times \boldsymbol{L}^{-1}\left(\bar{\mu}^{C} \mu^{D}+\mathcal{M}^{\prime \beta C} \mathcal{M}_{\beta}^{\prime D}\right) .
\end{aligned}
$$

(iii) The moduli space $\mathfrak{M}_{k, N}$ is not a smooth manifold: it has orbifold-type singularities that occur when $U(k)$ does not act freely. Physically these are points where an instanton shrinks to zero size, i.e. $w_{i u \dot{\alpha}}=0$ for a given $i$. We can illustrate this for the case of a single instanton in $S U(2)$. In this case, $\mathfrak{M}_{1,2}=\mathbb{R}^{4} \times \mathbb{R}^{4} / \mathbb{Z}_{2}$, where $\mathbb{R}^{4}$ corresponds to position of the instanton while the angular coordinates of the second $\mathbb{R}^{4}$ parameterize the $S U(2)$ gauge orientation and finally the radial coordinate of this factor is the scale 'size. It is important to emphasize that these singularities are not evidence of any sickness in the instanton calculus. In fact when calculating the instanton contribution to any physical quantity in field theory these short-distance singularities are prefectly harmless.

There is a natural way to smooth, or blow up, the singularities of $\mathfrak{M}_{k, N} \rightarrow \mathfrak{M}_{k, N}^{(\zeta)}$ : simply modify the ADHM constraints by adding a term proportional to the identity matrix to the righthand side:

$$
\mathcal{B}^{c} \equiv\left(\tau^{c}\right)_{\dot{\beta}}^{\dot{\alpha}}\left(\bar{w}^{\dot{\beta}} w_{\dot{\alpha}}+\bar{a}^{\prime \dot{\beta} \alpha} a_{\alpha \dot{\alpha}}^{\prime}\right)=\zeta^{c} 1_{[k] \times[k]} .
$$

The new term prevents any component $w_{u i \dot{\alpha}} \rightarrow 0$ and so instantons cannot shrink to zero size. For example, in the case $k=1$ and $N=2$ described 
above, it is possible to show that the orbifold factor $\mathbb{R}^{4} / \mathbb{Z}_{2}$ becomes the Eguchi-Hanson manifold. Remarkably, the smoothed moduli space $\mathfrak{M}_{k, N}^{(\zeta)}$ describes instantons in non-commutative gauge theory on a spacetime with non-commuting coordinates [17]:

$$
\left[x_{n}, x_{m}\right]=-i \bar{\eta}_{n m}^{c} \zeta^{c}
$$

where $\bar{\eta}_{n m}^{c}$ is a 't Hooft eta symbol.

\section{Meaning of ADHM}

This all seems rather mysterious: we have a curious set of data and an auxiliary $U(k)$ symmetry. The first point to make is that the ADHM constraints are generally intractable: one simply cannot find a solution for $k>3$. However, recently we found a way to solve the constraints for arbitrary $k$ when $N \geq 2 k$ as we describe later. The rather arcane ADHM construction can now be understood in two apparently different, althought intimately related, ways:

The Math Way. [18] The ADHM construction is an example of a hyper-Kähler quotient. One starts with $\mathbb{R}^{4 k N+4 k^{2}}$, which is naturally hyper-Kähler with a flat metric, and then imposes a triplet of constraints of the type (2.3). Finally one mods out by an auxiliary symmetry, in this case $U(k)$, leaving a hyper-Kähler manifold of dimension $4 k N$. It turns out that the measure on the ADHM moduli space $((\overline{2}-5.5)$ with $\mathcal{N}=$ 0 ) follows geometrically from this quotient construction as the measure induced on $\mathfrak{M}_{k, N}$ by the flat measure on $\mathbb{R}^{4 k N+4 k^{2}}$. Finally, the smoothed space described by the modified ADHM constraints $\left(12.133_{1}^{\prime}\right)$ is a natural deformation of the quotient construction which preserves the hyper-Kählarity.

The Physics Way. [13,19] The ADHM construction naturally arises in the dynamics of Dbranes in string theory. The low energy collective dynamics of $N$ coincident $\mathrm{D}(p+4)$-branes in Type II string theory is described by a $U(N)$ SUSY gauge theory in $p+5$-dimensions with 16 supercharges. An instanton in the world-volume theory of the $\mathrm{D}(p+4)$-branes is a soliton which has 4 transverse directions in the higher dimensional brane, i.e. is some kind of $p$-brane. The remarkable thing is that it is precisely a $\mathrm{D} p$ brane bound to the $\mathrm{D}(p+4)$-brane. In general $k \mathrm{D} p$-branes bound to the $N$ higher dimensional $\mathrm{D}(p+4)$-branes correspond to a charge $k$ instanton in a $U(N)$ SUSY gauge theory.

In order to see how this plays out, we have to consider the low energy collective dynamics of the $\mathrm{D} p$-branes. This is described by a SUSY $U(k)$ gauge theory with 16 supercharges, but with additional matter fields arising from the higher dimensional branes which break half of these supersymmetries. To be more specific, let us suppose that $p=3$. In this case a theory with $16 \mathrm{su}-$ percharges is $\mathcal{N}=4$ SUSY gauge theory. Let us analyse the spectrum of fields in terms of $\mathcal{N}=1$ supermultiplets. Along with the $\mathcal{N}=1$ vector multiplet containing the gauge field, there are 3 adjoint-valued chiral superfields $\Phi, X$ and $\tilde{X}$. The 6 real scalars of these chiral multiplets describe the transverse positions of the D3-branes and in particular $X$ and $\tilde{X}$ describe the positions of the D3-branes within the D7-branes, while $\Phi$ describes the separation between the D3- and D7-branes. Open string going between the D3branes and D7-branes give rise a $N$ chiral multiplets $Q$ and $\tilde{Q}$ in, respectively, the $\boldsymbol{k}$ and $\overline{\boldsymbol{k}}$ representations of the gauge group. The resulting theory has $\mathcal{N}=2$ supersymmetry and $X$ and $\tilde{X}$ form an adjoint hypermultiplet while $Q$ and $\tilde{Q}$ form $N$ fundamental hypermultiplets.

This gauge theory then describes the low energy dynamics of the D3-branes (in the presence of D7-branes). Let us consider the space of vacua of this theory. The theory has a Higgs branch where the gauge group is completely broken (the scalar components of) $\Phi=0$ and $Q, \tilde{Q}, X$ and $\tilde{X}$ are non zero. The equations describing the Higgs branch follow from the $D$ and $F$-flatness conditions and these precisely the ADHM constraints $(2.3)$ with the identifications

$$
w_{\dot{\alpha}}=\left(\begin{array}{c}
Q^{\dagger} \\
\tilde{Q}
\end{array}\right), \quad a_{\alpha \dot{\alpha}}^{\prime}=\left(\begin{array}{cc}
X^{\dagger} & \tilde{X} \\
-\tilde{X}^{\dagger} & X
\end{array}\right) .
$$

Hence there is a natural identification of $\mathfrak{M}_{k, N}$ and the Higgs branch of our $\mathcal{N}=2$ gauge theory. Notice that this gauge theory, with gauge group $U(k)$, is not the original $\mathcal{N}=4$ gauge theory that lives on the D7-branes, which has gauge group $U(N)$. The Higgs branch describes a situation in which the D3-branes lie inside the D7-branes $(\Phi=0)$. On the contrary the Coulomb branch, 
on which $Q=\tilde{Q}=0$, while $\Phi, X$ and $\tilde{X}$ are non zero, describes a situation in which the D3-branes have moved off the D7-branes. There are mixed branches which describe situations in which some of the D3-branes are on the D7-branes while some have moved off into the bulk. The points where $Q_{i}$ and $\tilde{Q}_{i}$ go to zero connect the different phases and correspond to points where the D3-branes can move off into the D7-branes. These are precisely the points where an instanton shrinks to zero size. So in a certain respect, that we will make explicit shortly, this stringy context leads to a certain resolution of the orbifold singularities of $\mathfrak{M}_{k, N}$.

However, there is more to this than an identification between $\mathfrak{M}_{k, N}$ and the Higgs branch of the gauge theory. If we dimensionally reduce the system to $p=-1$, so that we are describing a system of D-instantons and D3-branes, then the the partition function of the $U(k)$ gauge theory (which is now a 0-dimensional field - or matrixtheory) can be identified with the measure on the ADHM moduli space in the limit where bulk effects decouple from the branes, $\alpha^{\prime} \rightarrow 0$. The $4 \mathrm{D}$ gauge field and $\Phi$ can be amalgamated into $\chi_{a}$, $a=1, \ldots, 6$, an adjoint-valued 6 -vector. The bosonic part of the partition function is

$z_{k, N}=\int \frac{d^{4} a^{\prime} d^{2} w d^{6} \chi d^{3} D}{\operatorname{Vol} U(k)} \exp \left(-\operatorname{tr}_{k} \chi_{a} \boldsymbol{L} \chi_{a}\right.$
$\left.+\alpha^{\prime 4} \operatorname{tr}_{k}\left[\chi_{a}, \chi_{b}\right]^{2}-2 \alpha^{\prime 4} \operatorname{tr}_{k} D^{2}+i \operatorname{tr}_{k} D^{c} \mathcal{B}^{c}\right) \times \cdots$

Here, $D^{c}$ is an adjoint-valued 3 -vector that arises as an auxiliary field of the $4 \mathrm{D}$ theory. Now if we take $\alpha^{\prime}=0$, then the integral over $\chi_{a}$ is Gaussian and gives rise to a factor $(\operatorname{det} \boldsymbol{L})^{-3}$, while the $D^{c}$ are nothing but Lagrange multipliers for the ADHM constraints! Notice that the resulting partition function in this limit gives precisely the the bosonic parts of the measure on the ADHM moduli space in an $\mathcal{N}=4$ SUSY theory (2.5).

If we don't take the $\alpha^{\prime}=0$ limit, then in a sense we resolve the singularities of $\mathfrak{M}_{k, N}$ since the $D^{c}$ no longer act as Lagrange multipliers for the ADHM constraints, rather, the constraints are smeared over a scale $\sqrt{\alpha^{\prime}}$. How does this kind of resolution relate to the blow-up $\mathfrak{M}_{k, N}^{(\zeta)}$ ? The modifications of the ADHM constraints by the parameters $\zeta^{c}$ can naturally be incorporated into the stringy construction since they correspond to Fayet-Illiopolos (FI) couplings in the $U(k)$ gauge theory, i.e. add $-i \zeta^{c} \operatorname{tr}_{k} D^{c}$ to the exponent in (3.2). There are consequently two different ways to smooth $\mathfrak{M}_{k, N}$, via stringy corrections or via FI couplings; however, we shall argue later that they lead to the same effect.

Before we leave this section there are three further issues that we mention.

(i) It is important that $\chi_{a}$ also couples to a fermion bilinear:

$$
\Sigma_{A B}^{a} \operatorname{tr}_{k} \chi_{a}\left(\bar{\mu}^{A} \mu^{B}+\mathcal{M}^{\prime \alpha A} \mathcal{M}_{\alpha}^{\prime B}\right),
$$

for, when $\alpha^{\prime}=0$ and $\chi_{a}$ is integrated-out, a the 4 -fermion interaction ( $12 . \overline{1} \overline{2})$ is generated.

(ii) Hitherto, we have been considering the situation where the $N \mathrm{D}(p+4)$-branes are coincident; however, what happens when they separate? Consider the case with $p=-1$. From the point-of-view of the D3-branes the answer is straightforward: the scalars which correspond to the positions of the branes gain a $\operatorname{VEV}\left\langle\varphi_{a}\right\rangle$, a 6 -vector of $N \times N$ matrices, and one moves out onto the Coulomb branch of the $U(N)$ gauge theory. This effect is then easily incorporated into the D-instanton $U(k)$ theory, by modifying the following couplings:

$w_{\dot{\alpha}} \chi_{a} \rightarrow w_{\dot{\alpha}} \chi_{a}+\left\langle\varphi_{a}\right\rangle w_{\dot{\alpha}}, \mu^{A} \chi_{a} \rightarrow \mu^{A} \chi_{a}+\left\langle\varphi_{a}\right\rangle \mu^{A}$

These couplings have the form of mass terms for $w_{\dot{\alpha}}$. It turns out that the new couplings precisely reproduce the constrained instanton formalism [20] that describes instantons in theories with VEVs. The effect of the extra coupling to the VEVs is to suppress instantons of large size in the instanton measure and superconformal invariance is explicitly broken.

(iii) It is worth commenting on the case when $N=1$ and the original gauge theory has gauge group $U(1)$. It is well known that abelian theories do not have instantons; however, we can still define the ADHM construction. In this case, the ADHM constraints are explicitly solved by taking $w_{\dot{\alpha}}=0$ and $a_{n}^{\prime}=-\operatorname{diag}\left(X_{n}^{1}, \ldots, X_{n}^{k}\right)$. In other words, these "abelian instantons" are point like and moreover

$$
\mathfrak{M}_{k, 1}=\operatorname{Sym}^{k}\left(\mathbb{R}^{4}\right) .
$$


This space has singularities whenever 2 instantons coincide. However, one finds that the gauge potential that arises from the ADHM data is pure gauge. Nevertheless, when we modify the ADHM construction as in $(\overline{2} . \overline{1} \overline{1})$, in other words consider instantons in a non-commutative theory, then the abelian instanton solutions become non-trivial. The deformed space $\mathfrak{M}_{k, 1}^{(\zeta)}$ is smooth; for example, $\mathfrak{M}_{2,1}=\mathbb{R}^{4} \times \mathbb{R}^{4} / \mathbb{Z}_{2}$, while the deformation replaces the orbifold factor with the Eguchi-Hanson manifold. So we see $\mathfrak{M}_{1,2}=\mathfrak{M}_{2,1}$ and $\mathfrak{M}_{1,2}^{(\zeta)}=\mathfrak{M}_{2,1}^{(\zeta)}$, a property that does not generalize to $N>1$ and $k>2$.

\section{Calculations with Instantons}

In this section we shall summarize a number of interesting applications of the instanton calculus. We will primarily be interested in the $\mathcal{N}=4$ theory with possible stringy corrections, FI couplings and VEVs.

\subsection{The AdS/CFT correspondence}

The AdS/CFT correspondence realizes the old idea that string theory describes large- $N$ of gauge theory [21]. In fact it is much stronger: $\mathcal{N}=4$ SUSY gauge theory is equivalent to Type IIB string theory compactified on $A d S_{5} \times S^{5}$. As usual with a duality it is hard to prove, since calculations can only be done at weak coupling in the gauge theory, $g^{2} N \ll 1$, while - presently at least - calculations on the string theory side can only be done in the classical supergravity limit where the radius of curvature $R \gg \sqrt{\alpha^{\prime}}$; which means $g^{2} \ll 1$ while $g^{2} N \gg 1$. Some quantities, however, are protected against renormalization in $g^{2} N$, and the value calculated in the gauge theory can be compared directly to the value extracted from the supergravity approximation to string theory.

For us the relevant correlation functions involve 16 dilatinos $\Lambda$ on the supergravity side that correspond to a certain composite operator in the gauge theory. These correlation functions receive contributions from $\mathrm{D}$-instantons in the string theory whose coupling dependence singles them out as instanton contributions in the gauge theory. The correspondence requires that the $k$ instanton contribution to the correlator, in the infra-red and at leading order in $\frac{1}{N}$, should be, schematically, [22, 23]

$$
\begin{gathered}
\left\langle\Lambda\left(x_{1}\right) \cdots\right. \\
\left.\times \int\left(x_{16}\right)\right\rangle \sim \sqrt{N} g^{-24} q^{k} k^{25 / 2} \sum_{d \mid k} d^{-2} \\
\rho^{5} \\
\times \int \frac{d^{4} X d \rho}{16} \mathcal{F}\left(x_{i}-X, \rho\right) .
\end{gathered}
$$

Here, $\left\{X_{n}, \rho\right\}$ parameterizes a point in $A d S_{5}$ and the details of the expression for the integrand may be found in [13]. What is remarkable about $(4.1)$ is that the $k$ dependence is only through the numerical pre-factor $q^{k} k^{25 / 2} \sum_{d \mid k} d^{-2}$. This looks like a disaster because there seems little chance that the integral over $k$ instantons, with its intrinsic complexity, would reduce to something that is simply a number times a one instanton contribution.

The $k$-instanton contribution to the correlators involves inserting into the measure, (2.5i) with $\mathcal{N}=4$ along with the 4 -fermion coupling $(2 . \overline{1} \overline{1})$, the 16 composite operators. This contribution turns out to be calculable at leading order in $1 / N$ in a way that we summarize below [13]:

(i) For $N \geq 2 k$, and so certainly at large $N$, the ADHM constraints can be solved by a simple change of variables: the biggest impediment to progress with the ADHM construction proves to be entirely benign. The idea involves changing variables from the $w_{\dot{\alpha}}$ to quadratic gauge invariant variables

$$
W_{\dot{\beta}}^{\dot{\alpha}}=\bar{w}^{\dot{\alpha}} w_{\dot{\beta}}
$$

The ADHM constraints are then linear in $W_{\dot{\beta}}^{\dot{\alpha}}$, as is apparent from (2.3), and the the $\delta$-function constraints in $(2.5)$ may trivially be solved.

(ii) The 4-fermion term in the instanton action $(\overline{2} \overline{1} \overline{2})$ can be bilinearized by introducing a 6 -vector of $U(k)$-adjoint variables $\chi_{a}$. The Grassmann collective coordinates can then integratedout. The $\chi_{a}$ variables are precisely those that arise naturally in the D-instanton/D3-brane system described previously.

(iii) The remaining expression is then amenable to a saddle-point approximation at large $N$. The saddle-point solution has a very simple interpretation. Each of the $k$ instantons are embedded 
in mutually commuting $S U(2)$ subgroups of the gauge group, as one might have expected on statistical grounds alone. Furthermore, and less intuitive, is that they have the same size $\rho$ and sit at the same point $X_{n}$ in spacetime; so at the saddle point

$$
\bar{w}^{\dot{\alpha}} w_{\dot{\beta}}=\rho^{2} \delta_{\dot{\beta}}^{\dot{\alpha}} 1_{[k] \times[k]}, \quad a_{n}^{\prime}=-X_{n} 1_{[k] \times[k]} .
$$

Furthermore, the auxiliary variables $\chi_{a}$ have the saddle-point value

$$
\chi_{a}=\rho^{-1} \hat{\Omega}_{a} 1_{[k] \times[k]},
$$

where $\hat{\Omega}_{a}$ is a unit 6 -vector. So the saddle point is parameterized by a point in $A d S_{5} \times S^{5}$ ! Amazingly, instantons in the gauge theory act as a probe that feel the ten-dimensional geometry of the dual theory. Notice that the $S^{5}$ part of the geometry arises from the auxiliary variables $\chi_{a}$.

(iii) The integral of the fluctuations around the saddle-point solution assembles into something that is known: precisely the partition function of $\mathcal{N}=110 \mathrm{D} S U(k)$ Yang-Mills dimensionally reduced to 0 dimensions, where the $10 \mathrm{D}$ gauge field is formed from the traceless parts of $a_{n}^{\prime}$ and $\chi_{a}$. This is known to be proportional to $\sum_{d \mid k} d^{-2}[24,25]$.

Putting all of this together immediately solves the puzzle alluded to above: any correlation function will look one instanton-like up to an overall $k$ dependent factor. In addition, one can show that the $k$-dependence and overall factor of $\sqrt{N}$ are exactly reproduced. Instantons consequently provide one of the most convincing pieces of evidence in favour of the AdS/CFT correspondence.

We can also couch our result in terms of the partition function of the D-instanton/D3-brane system:

$$
\begin{aligned}
& z_{k, N} \underset{N \rightarrow \infty}{=} 2^{3-2 k} \pi^{6 k-25 / 2} \\
& \sqrt{N} k^{3 / 2} \sum_{d \mid k} d^{-2} \int \frac{d^{4} X d \rho d^{5} \hat{\Omega}}{\rho^{5}} \cdot d^{8} \xi d^{8} \bar{\eta}
\end{aligned}
$$

where $X$ and $\rho$ are the overall position and scale size, respectively, while $\xi$ and $\bar{\eta}$ are the 8 SUSY and superconformal fermion zero modes, respectively. It is possible to generalize these kinds of calculations to other AdS/CFT duals [26].

\subsection{Instanton effects in D3-branes}

The collective excitations of $N$ D3-branes are described at low energies by an $\mathcal{N}=4$ SUSY gauge theory with gauge group $U(N)$. However, the minimal $\mathcal{N}=4$ Lagrangian is only valid at low energy and there is an infinite tower of the higher derivative interactions that come with powers of $\alpha^{\prime}$, the string length scale. Some of these, but not all are encoded in the Born-Infeld Lagrangian. In [27], it was argued that in the case of a single D3-brane, instantons contribute to certain terms of order $\alpha^{4}$, including one of the form $(\partial F)^{4}$, where $F$ is the abelian field strength. Furthermore, the $S L(2, \mathbb{Z})$ modular symmetry of the Type IIB string theory, which is realized as electro-magnetic duality in the D3-brane theory, fixes the instanton contributions exactly. In fact the coupling to this term in the effective action involves the logarithm of the Dedekind eta function:

$$
\ln \left|\eta(\tau)^{4}\right|=-\frac{\pi}{3} \operatorname{Im} \tau-2 \sum_{k=1}^{\infty}\left(q^{k}+\bar{q}^{k}\right)\left[\sum_{d \mid k} d^{-1}\right] .
$$

Here, the first term is a tree-level contribution, while the other terms come from $k$ instantons and $k$ anti-instantons, respectively.

We can relate the $k$-instanton terms in the effective action of the D3-brane predicted by Green and Gutperle [27] for the case $N=1$ (without FI and VEV terms) to the $k$-instanton partition function modded out by the integral over the overall $k$-instanton position in $\mathbb{R}^{4}$ and its superpartners (the 8 supersymmetric fermion zero modes)

$$
\widehat{z}_{k, 1}\left(\zeta, \alpha^{\prime}\right)=\sum_{d \mid k} d^{-1}
$$

Here, the FI coupling $\zeta$, absent at the start, arises as a source.

What is interesting about the string result (4.7) is that in order to have a non-trivial contribution when $\zeta=0$, it is absolutely essential to have the $\alpha^{\prime}$ corrections in the D-instanton/D3brane system. Another way of seeing this is that superconformal invariance must be broken. It turns out that when $\zeta \neq 0$ we can legitimately 
set $\alpha^{\prime}=0$ to yield:

$$
\widehat{z}_{k, 1}(\zeta, 0)=\sum_{d \mid k} d^{-1}
$$

which is then a statement about the integral over the resolved centered moduli space $\widehat{\mathfrak{M}}_{k, 1}^{(\zeta)}$, where $\mathfrak{M}_{k, N}^{(\zeta)}=\mathbb{R}^{4} \times \mathfrak{M}_{k, N}^{(\zeta)}$. Note that the integral (4.7i) does not actually depend on the $\alpha^{\prime}$ coupling; a fact that we shall return to in

The whole story of D-instanton corrections to the D3-brane effective action generalizes to the non-abelian case of $N$ D3-branes [28]. In this case, it is necessary that the D3-branes are separated by adding VEVs $\langle\varphi\rangle$ so that theory is in a Coulomb phase. In this case, there is generalization of (4.7):

$$
\widehat{z}_{k, N}\left(\zeta, \alpha^{\prime},\langle\varphi\rangle\right)=N \sum_{d \mid k} d^{-1}
$$

It is possible to prove this using Morse theory arguments. First of all, one can set $\alpha^{\prime}$ to zero in (4.9i). If the VEVs vanished, the latter quantity is Gauss-Bonnet-Chern integral on $\widehat{\mathfrak{M}}_{k, N}^{(\zeta)}$ (see the next section). Turning on the VEV has the effect of introducing a Morse function on $\widehat{\mathfrak{M}}_{k, N}^{(\zeta)}$ and using standard arguments the integral $\widehat{z}_{k, N}(\zeta, 0,\langle\varphi\rangle)$ localizes onto the critical point set. These are the submanifold of $\mathfrak{M}_{k, N}^{(\zeta)}$ where

$$
w_{\dot{\alpha}} \chi_{a}+\left\langle\varphi_{a}\right\rangle w_{\dot{\alpha}}=0
$$

The VEV $\left\langle\varphi_{a}\right\rangle$ is a 6-vector of diagonal $N \times N$ matrices. The critical points correspond to associating each instanton with a particular D3brane; in other words a partition $k=\left\{k_{1}, \ldots, k_{n}\right\}$, where $k_{u} \geq 0$. For each partition the critical point set is a product of abelian, $N=1$, instanton moduli spaces:

$$
\mathfrak{M}_{k_{1}, 1} \times \cdots \times \mathfrak{M}_{k_{n}, 1}
$$

Localizing the integral on the critical point sets gives us a relation of the form

$z_{k, N}(\zeta, 0,\langle\varphi\rangle)=\sum_{\left\{k_{j}\right\}} z_{k, 1}(\zeta, 0) \times \cdots \times z_{k, 1}(\zeta, 0)$.

Notice that we have not separated out the centerof-mass integrals yet. Each factor $z_{k, 1}(\zeta, 0)$ leaves
8 unsaturated Grassmann integrals; hence, most of the partitions in the sum have more than 8 unsaturated Grassmann integrals and will not contribute to $\widehat{z}_{k, N}(\zeta, 0,\langle\varphi\rangle)$. Only the partitions where all $k$ of the instantons live on the same D3-brane will survive, and there are $N$ of these; so

$$
\widehat{z}_{k, N}(\zeta, 0,\langle\varphi\rangle)=N \widehat{z}_{k, 1}(\zeta, 0)=N \sum_{d \mid k} d^{-1}
$$

What is striking about this result is that for $N>$ 1 it holds only for nonvanishing VEVs, i.e. in the Coulomb phase, but nevertheless the right-hand side of $\left({ }_{-}^{-} . \bar{g}\right)$ is independent of $\langle\varphi\rangle$.

\subsection{The Euler Characteristic of $\widehat{\mathfrak{M}}_{k, N}$}

This section investigates the relation between various physical quantities extracted from the brane system and the Euler characteristic of instanton moduli space [28]. The Euler characteristic $\chi$ of resolved (centered) moduli space $\widehat{\mathfrak{M}}_{k, N}^{(\zeta)}$ was deduced from the Morse theory analysis of Nakajima [29] (see also [30]):

$$
1+\sum_{k=1}^{\infty} \chi\left(\widehat{\mathfrak{M}}_{k, N}^{(\zeta)}\right) q^{k}=\frac{1}{\prod_{j=1}^{\infty}\left(1-q^{j}\right)^{N}} .
$$

Notice that the generating function above is, up to a factor of $q^{-N / 24}, \eta^{-N}$, where $\eta$ is the Dedekind eta-function and, as such, has interesting modular properties. In fact there is a very intriguing more general relation between the generating functions of the Euler characteristics for instantons in gauge theories defined on different 4 manifolds, and the characters of 2-dimensional conformal field theories [31]. The question is how this relates to the the resulting partition function of the D-instanton/D3-brane system, with the centre-of-mass and the 8 SUSY zero modes factored off, denoted

$$
\widehat{z}_{k, N}\left(\zeta, \alpha^{\prime},\langle\varphi\rangle\right) \text {. }
$$

where the dependence on FI couplings, VEVs and $\alpha^{\prime}$ is indicated.

Conventional wisdom suggests that the quantity $\widehat{z}_{k, N}$ should yield the Euler characteristic of $\widehat{\mathfrak{M}}_{k, N}$ as we shall now explain. The point is that the measure over the instanton moduli space in 
an $\mathcal{N}=4$ gauge theory has the 4 -fermion term $(12 . \overline{1} \overline{1})$. The 4-index tensor that appears is nothing but the Riemann tensor of $\widehat{\mathfrak{M}}_{k, N}$ and saturating the fermion integrals from the action brings down powers of the Riemann tensor contracted in such a way that the resulting integral over $\widehat{\mathfrak{M}}_{k, N}$ is precisely the Gauss-Bonnet-Chern (GBC) integral. On a compact manifold this would give the Euler characteristic. However, our manifold is non-compact and in this case the GBC integral only gives the bulk contribution to the Euler characteristic and there can be additional boundary contributions arising from either the boundary at infinity or from the orbifold singularities.

One immediate problem with our integral is that due to superconformal invariance the integral over the overall scale size of the configuration is divergent. In addition the integrals over the 8 superconformal zero modes are not saturated by any fermionic insertions. This is evident from our large- $N$ expression for $z_{k, N}$ in $(4.5)$. The way to deal with this is to break superconformal invariance by blowing up the singularities of $\widehat{\mathfrak{M}}_{k, N}$ to give the smooth, but still non-compact, space $\widehat{\mathfrak{M}}_{k, N}^{(\zeta)}$. This is achieved by including the FI couplings $\zeta$. The integral over the scale size is now rendered convergent and the 8 superconformal zero modes are lifted. Factoring off the integrals over the overall position and 8 SUSY zero modes, gives the GBC integral in the large $N$ limit:

$\widehat{z}_{k, N}(\zeta, 0,0) \underset{N \rightarrow \infty}{=} 2^{3-2 k} \pi^{6 k-13 / 2} \sqrt{N} k^{3 / 2} \sum_{d \mid k} d^{-2}$.

We can also calculate the $k=1$ case with arbitrary $N$ exactly [28]:

$$
\widehat{\varkappa}_{1, N}(\zeta, 0,0)=\frac{2^{1-2 N}(2 N) !}{N !(N-1) !} .
$$

These expressions are not integers and there must be contributions coming form the boundary at infinity. In particular, on the basis of the formula $(\bar{A} . \overline{1} \overline{4})$, we expect $\chi\left(\widehat{\mathfrak{M}}_{1, N}^{(\zeta)}\right)=N$. For $k=1$ and $\bar{N}=2$ we can calculate the surface contribution and show how the $(\bar{A} \overline{1})$ calculation is consistent with $(\overline{4} \cdot \overline{1} \overline{7})$. In this case $\widehat{z}_{1,2}(\zeta, 0,0)=\frac{3}{2}$, while $\chi=2$. Recall that the singular space $\widehat{\mathfrak{M}}_{1,2}=\mathbb{R}^{4} / \mathbb{Z}_{2}$ while the blow-up $\widehat{\mathfrak{M}}_{1,2}^{(\zeta)}$ is the
Eguchi-Hanson manifold. In [32], the authors calculate the Euler characteristic of this space and show that it receives a bulk contribution of $\frac{3}{2}$, matching ( $\left.\bar{A} . \overline{1} \overline{7}\right)$, and-importantly for us-a boundary contribution of $\frac{1}{2}$.

For the case $k=1$ at least (and arbitrary $N$ ) one can alter the asymptotic behaviour of the GBC integral so that there is no contribution from infinity and the bulk integral yields directly the Euler characteristic $\chi=N$. This modification involves moving onto the Coulomb branch of the $\mathcal{N}=4$ theory by introducing VEVs as in $(3.4)$. We then find by explicit calculation [28]

$$
\widehat{z}_{1, N}(\zeta, 0,\langle\varphi\rangle)=N
$$

Interestingly, moving out onto the Coulomb branch can be interpreted as introducing a potential on the moduli space. This potential has precisely $N$ critical points whose number saturates the value of the integral as one might have expected from standard Morse theory arguments. In fact, in a similar way for general $k$, there is a more refined potential that arises from giving all the fields twisted masses [28], which lifts all the flat directions and has a number of isolated critical points which yields directly the result of Nakajima [29]. To complete the story of $k=1$ we can also calculate explicitly

$$
\begin{gathered}
\widehat{z}_{1, N}(0,0,\langle\varphi\rangle)=N-\frac{2^{1-2 N}(2 N) !}{N !(N-1) !} \\
\equiv \widehat{z}_{1, N}(\zeta, 0,\langle\varphi\rangle)-\widehat{z}_{1, N}(\zeta, 0,0) .
\end{gathered}
$$

It remains to be seen whether similar relations hold for arbitrary $k$; however, we feel that it should be possible to relate $\widehat{z}_{k, N}(\zeta, 0,\langle\varphi\rangle)$ and $\widehat{z}_{k, N}(0,0,\langle\varphi\rangle)$ by investigating the behaviour of the integrals near the singularities. The quantity $\widehat{\mathcal{Z}}_{k, N}(0,0,\langle\varphi\rangle)$ is particularly interesting because it appears in the mismatch between the microscopic couplings and the effective couplings in Seiberg-Witten theory of the mass deformed $\mathcal{N}=4$ theory [33].

Up till now, we have not considered the effect of the stringy couplings. Actually, turning on the $\alpha^{\prime}$ couplings has no effect; in other words,

$$
\widehat{z}_{k, N}\left(\zeta, \alpha^{\prime},\langle\varphi\rangle\right)=\widehat{z}_{k, N}(\zeta, 0,\langle\varphi\rangle) \text {. }
$$

as long as either $\zeta$ or $\langle\varphi\rangle$ are non-vanishing so that the right-hand side is well defined. We have 
already seen in (4.9i) this is implied by a generalization of the Green-Gutperle analysis to $N>$ 1. There is another way to motivate this result from completely different considerations involving ideas of Cohomological Field Theory [24]. In a very small nut-shell, the integral $\widehat{z}_{k, N}\left(\zeta, \alpha^{\prime},\langle\varphi\rangle\right)$ has a nilpotent BRST-type symmetry derived from its supersymmetry. The $\alpha^{\prime}$ couplings can be shown to be $Q$-exact and hence can be set to 0 , as long as the resulting integral is well defined.

There is clearly a lot of interesting relations between the Euler characteristic of instanton space and various physical quantities in the gauge/string theory that remain to be uncovered.

We would like to thank Stefan Vandoren for conversations and collaboration and our erstwhile collaborator Michael Mattis. This work was supported by a TMR network, EC contract number FMRX-CT96-0012.

\section{References}

[1] V.A. Novikov, M.A. Shifman, A.I. Vainshtein and V.I. Zakharov, Nucl. Phys. B229 (1983) 407

D. Amati, K. Konishi, Y. Meurice, G.C. Rossi and G. Veneziano, Phys. Rept. 162 (1988) 169

[2] T. J. Hollowood, V. V. Khoze, W. Lee and M. P. Mattis, Nucl. Phys. B570 (2000) 241 [hep-th/9904116].

[3] I. Affleck, M. Dine and N. Seiberg, Phys. Rev. Lett. 51 (1983) 1026; Nucl. Phys. B241 (1984) 493.

V.A. Novikov, M.A. Shifman, A.I. Vainshtein and V.I. Zakharov, Nucl. Phys. B260 (1985) 157.

[4] N. M. Davies, T. J. Hollowood, V. V. Khoze and M. P. Mattis, Nucl. Phys. B559 (1999) 123 [hep-th/9905015]

N. M. Davies, T. J. Hollowood and V. V. Khoze, hep-th/0006011

[5] K. Lee and P. Yi, Phys. Rev. D56 (1997) 3711 [hep-th/9702107].

[6] S. Katz and C. Vafa, Nucl. Phys. B497 (1997) 196 [hep-th/9611090].

C. Gomez and R. Hernandez, Nucl. Phys. Proc. Suppl. 61A (1998) 172 [hep-th/9703036].

[7] N. Dorey, JHEP $\mathbf{9 9 0 7} \quad$ (1999) 021 [hep-th/9906011].
[8] O. Aharony, N. Dorey and S. P. Kumar, JHEP 0006 (2000) 026 [hep-th/0006008] N. Dorey and S. P. Kumar, JHEP 0002 (2000) 006 [hep-th/0001103].

[9] I. Jack, Nucl. Phys. B174 (1980) 526 B. Berg and M. Luscher, Nucl. Phys. B160 (1979) 281.

[10] M.F. Atiyah, N.J. Hitchin, V.G. Drinfeld and Y.I. Manin, Phys. Lett. 65A (1978) 185.

[11] E. Corrigan, D. Fairlie, P. Goddard and S. Templeton, Nucl. Phys. B140 (1978) 31.

[12] V.V. Khoze, M.P. Mattis and M.J. Slater, Nucl. Phys. B536 (1998) 69 [hep-th/9804009].

[13] N. Dorey, T.J. Hollowood, V.V. Khoze, M.P. Mattis and S. Vandoren, Nucl. Phys. B552 (1999) 88 [hep-th/9901128], JHEP 06 (1999) 023 [hep-th/9810243].

[14] H. Osborn, Ann. of Phys. 135 (1981) 373.

[15] N. Dorey, V.V. Khoze and M.P. Mattis, Phys. Rev. D54 (1996) 2921 [hep-th/9603136]; Phys. Rev. D54 (1996) 7832 [hep-th/9607202].

[16] N. Dorey, V.V. Khoze and M.P. Mattis, Nucl. Phys. B513 (1998) 681 [hep-th/9708036]

N. Dorey, T.J. Hollowood, V.V. Khoze and M.P. Mattis, Nucl. Phys. B519 (1998) 470 [hep-th/9709072].

[17] N. Nekrasov and A. Schwarz, Commun. Math. Phys. 198 (1998) 689 [hep-th/9802068].

[18] N. J. Hitchin, A. Karlhede, U. Lindstrom and M. Rocek, Commun. Math. Phys. 108 (1987) 535.

[19] M. Douglas, hep-th/9512077, hep-th/9604198 E. Witten, Nucl. Phys. B460 (1996) 541, hep-th/9511030.

[20] I. Affleck, M. Dine and N. Seiberg, Phys. Rev. Lett. 51 (1983) 1026; Nucl. Phys. B241 (1984) 493.

[21] O. Aharony, S. S. Gubser, J. Maldacena, H. Ooguri and Y. Oz, Phys. Rept. 323 (2000) 183 [hep-th/9905111].

[22] T. Banks and M.B. Green, JHEP 05:002 (1998) [hep-th/9804170]

[23] M. Bianchi and S. Kovacs, hep-th/9811060 M. Bianchi, M. B. Green, S. Kovacs and G. Rossi, JHEP $9808 \quad$ (1998) 013 [hep-th/9807033]. 
[24] G. Moore, N. Nekrasov and S. Shatashvili, Commun. Math. Phys. $209 \quad$ (2000) 77 [hep-th/9803265].

[25] W. Krauth, H. Nicolai and M. Staudacher, Phys. Lett. B431 (1998) 31, hep-th/9803117 W. Krauth and M. Staudacher, Phys. Lett. B435 (1998) 350 , hep-th/9804199

[26] T. J. Hollowood, V. V. Khoze and M. P. Mattis, hep-th/9910118

T. J. Hollowood, JHEP 9911 (1999) 012 [hep-th/9908201]

T. J. Hollowood and V. V. Khoze, Nucl. Phys. B575 (2000) 78 [hep-th/9908035]

E. Gava, K. S. Narain and M. H. Sarmadi, Nucl. Phys. B569 (2000) 183 [hep-th/9908125].

[27] M. B. Green and M. Gutperle, hep-th/0002011.

[28] N. Dorey, T.J. Hollowood and V.V. Khoze, to appear.

[29] H. Nakajima, In *Sanda 1993, Topology, geometry and field theory* 129-136.

[30] O. Aharony, M. Berkooz and N. Seiberg, Adv. Theor. Math. Phys. 2 (1998) 119 [hep-th/9712117].

[31] C. Vafa and E. Witten, Nucl. Phys. B431 (1994) 3 [hep-th/9408074].

[32] T. Eguchi, P. B. Gilkey and A. J. Hanson, Phys. Rept. 66 (1980) 213.

[33] N. Dorey, V. V. Khoze and M. P. Mattis, Phys. Lett. B396 (1997) 141 [hep-th/9612231]. 